\title{
Absorbing boundary conditions for Schrödinger equation in a time-dependent interval
}

\author{
O. Karpova ${ }^{1,2}$, K. Sabirov ${ }^{1,3}$, D. Otajanov ${ }^{1}$, A. Ruzmetov ${ }^{1}$, A. A. Saidov ${ }^{1}$ \\ ${ }^{1}$ Turin Polytechnic University in Tashkent, \\ 17 Kichik Halka Yuli, Tashkent, 100095, Uzbekistan \\ ${ }^{2}$ National University of Uzbekistan, Tashkent, Uzbekistan \\ ${ }^{3}$ Tashkent University of Information Technologies, Tashkent, Uzbekistan \\ ola_july@mail.ru
}

PACS 02.60.Lj; 02.70.Bf

DOI 10.17586/2220-8054-2017-8-1-13-19

We consider the reflection-transmission of the Gaussian wave packet through the moving wall with absorbing boundary conditions based on the time dependent one-dimensional Schrödinger equation. The reflection coefficient is calculated for the case when the walls are fixed, and probability density is calculated for the case when the wall is moving linearly.

Keywords: absorbing boundary conditions, Gaussian wave packet, one-dimensional Schrödinger equation.

Received: 10 August 2016

Revised: 5 September 2016

\section{Introduction}

Absorbing boundary conditions $(\mathrm{ABC})$ for different wave equations have attracted much attention in different practically important contexts (see, e.g., papers [1-10] for review). Such boundary conditions describe absorption of particles and waves in their transition from one domain to another one. From the physical viewpoint particle absorption can occur in dissipative media. In addition, such type of boundary condition appears in reflectionless transmission of particles (waves) through the boundary of a given domain. Therefore one uses similar terminology for the boundary conditions of both types of processes, calling them absorbing or transparent boundary conditions. Strict mathematical treatment of $\mathrm{ABC}$ for different wave equations, including quantum mechanical Schrödinger equation can be found in the Refs. [11-16]. For both processes the boundary conditions can be derived by factorization of the differential operator corresponding to a wave equation which in general lead to complicated equations for the boundary conditions The explicit form of such boundary conditions is much more complicated than those of Dirichlet, Neumann and Robin conditions.

For absorbing boundary conditions the wave equation cannot be solved analytically and always requires using numerical methods. Depending on the type of the wave equation, they require different discretization schemes, which vary also for the process types. Finding an effective numerical scheme is a complicated task, especially, for quantum mechanical wave equations. Therefore, in some cases it is reasonable to use approximate methods enabling simplification of the numerical solution. One such method, based on the use of dispersion relation was introduced in the Ref. [11] for solving time-dependent Schrödinger equation with ABC on a finite interval. Later, it was improved by Kuska [11] with the use of alternative approximation for dispersion relation. In this paper we extended the results of Kuska to the case of moving boundaries. In particular, using the approach developed in the Ref. [12], we reduce time-dependent Schrödinger equation with moving boundary conditions into that of fixed ones. Imposing the $\mathrm{ABC}$ for the problem with moving boundaries, we derive the corresponding boundary conditions for the Schrödinger equation on a finite interval with unit length which is then solved numerically.

\section{Absorbing boundary conditions for fixed interval}

Here, following the Refs. $[10,11]$ we briefly recall derivation of the approximate $\mathrm{ABC}$ for one dimensional Schrödinger equation on a finite interval. Consider the one-dimensional time-dependent Schrödinger equation:

$$
i \hbar \frac{\partial \psi}{\partial t}(x, t)=-\frac{\hbar^{2}}{2 m} \frac{\partial^{2} \psi}{\partial x^{2}}(x, t)+V(x) \psi(x, t)
$$

describing the motion of a quantum particle with the mass $m$ in the interval $x \in(-\infty,+\infty)$ under the influence of the potential $V$. Taking a plane wave of the form:

$$
\psi(x, t)=\exp [-i(\omega t-k x)]
$$


from Eqs. (1) and (2) one gets the dispersion relation for the wave vector $k$ [11]:

$$
\hbar^{2} k^{2}=2 m[\hbar \omega-V] .
$$

This relation can be solved for $k$ and yields (in the units $m=\hbar=1$ ):

$$
k= \pm \sqrt{2[\omega-V]},
$$

where the plus sign describes waves moving to $x=+\infty$ and the minus sign means waves moving to $x=-\infty$. The left boundary has to be transparent for waves travelling to the left and the right boundary must be transparent for those heading right.

The square root function can be approximated using the rational function approximation method following [11]:

$$
\sqrt{z-z_{0}} \approx \sqrt{z_{0}} \frac{1+3 z / z_{0}}{3+z / z_{0}} .
$$

With the approximation (5) for the square root in the dispersion relation (4) one gets:

$$
k= \pm k_{0} \sqrt{\frac{2(\omega-V)}{k_{0}^{2}}} \approx \pm k_{0} \frac{1+3 z}{3+z},
$$

with

We write (6) as:

$$
z=\frac{2(\omega-V)}{k_{0}^{2}} .
$$

$$
k(3+z) \approx \pm k_{0}(1+3 z) .
$$

Putting expression for $z$ into the above equation, one gets:

$$
k\left(3 \frac{k_{0}^{2}}{2}+\omega-V\right) \approx \pm k_{0}\left(\frac{k_{0}^{2}}{2}+3 \omega-3 V\right) .
$$

By using the correspondence between quantities $\mathrm{k}$ and $\omega$ with their operator definitions:

$$
k \Leftrightarrow-i \frac{\partial}{\partial x} \quad \text { and } \quad \omega \Leftrightarrow i \frac{\partial}{\partial t},
$$

we obtain the following partial differential equation for the $\mathrm{ABC}$ :

$$
-i\left(3 \frac{k_{0}^{2}}{2}-V\right) \frac{\partial \psi}{\partial x}(x, t)+\frac{\partial^{2} \psi}{\partial t \partial x}(x, t)= \pm k_{0}\left(\frac{k_{0}^{2}}{2}-3 V\right) \psi(x, t) \pm 3 i k_{0} \frac{\partial \psi}{\partial t}(x, t) .
$$

The plus sign in (7) corresponds to the boundary conditions for the right side wall of the interval, while the minus sign in (7) corresponds to the boundary conditions for the left wall of the interval.

\subsection{Numerical results}

For the numerical experiments, we consider the transport of Gaussian wave packet:

$$
\psi(x, 0)=\sqrt{\pi \sigma} \exp \left(-\frac{(x-\xi)^{2}}{2 \sigma}\right) \exp \left(i k_{0} x\right)
$$

through the wall located at the right side. We consider that the particle is initially located near the right wall $(\xi=3 L / 4)$ in order to minimize the calculation time of the reflection-transmission process through this wall. The discrete one dimensional space is chosen between $-2 L<x<4 L$, which is large enough to satisfy the normalization condition:

$$
\int_{-\infty}^{+\infty} \psi^{*}(x, t) \psi(x, t) d x=1
$$

during numerical computation.

In Fig. 1, the evolution of the Gaussian wave packet described by the time-dependent Schrödinger equation with ABC given by Eq. (7) is presented. As it can be seen from this plot, for given initial conditions of the Gaussian wave packet, the reflectionless transmission through the boundary of the domain occurs.

In Fig. 2, we plot the dependence of the reflection coefficient on the initial mean wave vector of the wave packet. As it can be seen from the plot, there is an energy range for which the reflection coefficient becomes minimal. For these energies the wall becomes almost transparent. In order to calculate the reflection coefficient, $R$, we follow the evolution of the initially normalized probability of the quantum particle (wave packet) inside the interval $(x \in[0, L])$ and continue this until that moment when the scattering starts through the domain wall 


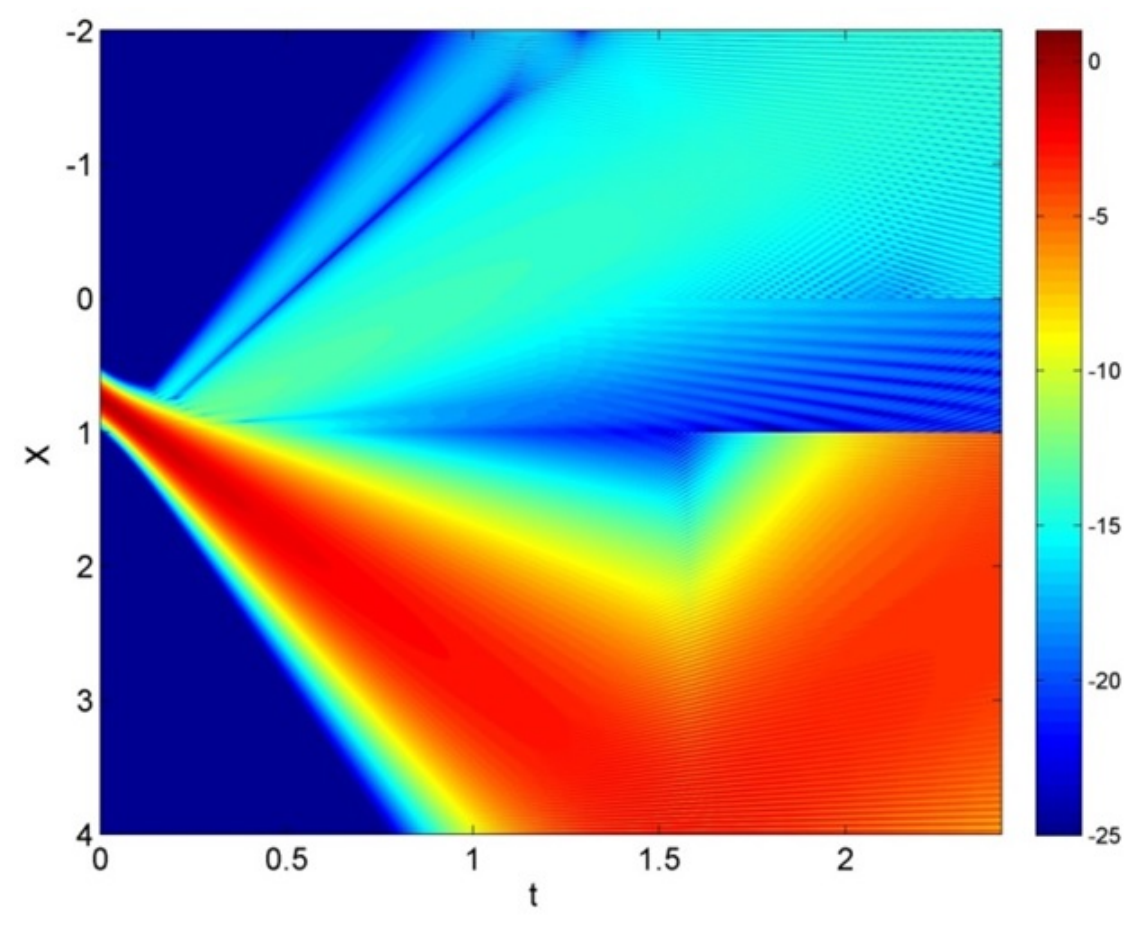

FIG. 1. (Color online) The spatiotemporal evolution of the probability density in logarithmic scale for the Gaussian wave packet described by Eq. (1), for which absorbing boundary conditions (7) are imposed. Initial wave packet width $\sigma=0.05 L$, the length of the interval $L=1$, and the initial position of quantum particle (wave packet) is $\xi=3 L / 4$. Numerical calculations are carried out with the discretization parameters: one dimensional space grid size $\Delta x=10^{-3}$ and time step size $\Delta t=10^{-4}$. The mean value of the wave vector of the initial wave packet is $k_{0}=25$.

(at $x=L$ ) with $\mathrm{ABC}$, and to the time when the scattering (reflection and transmission) processes are totally completed. Normalization condition implies that the summation of the reflection and transmission coefficient must be equal to 1, until the domain (discretized 1D space) is taken long enough $(-2 L<x<4 L)$ where the tail of probability density of the wave packet will not reach to the edge of the domain $($ at $x=4 L)$ due to dispersion during simulation.

For the normalized wave function by Eq. (9), one can easily track of the transmitted quantum particle (wave packet) position by computing the following integral:

$$
\int_{L}^{4 L} \psi^{*}(x, t) x \psi(x, t) d x,
$$

which is necessary to verify that the transmission was completed totally.

Easy quantification of the wave packet reflection through the $\mathrm{ABC}$ can be done by checking the probability of the wave packet inside the domain $-2 L<x<4 L$. During the transmission process, the integral (10) will be increasing and when it reaches to some value and stabilizes at this value, one can be sure that the process is completed.

\section{Absorbing boundary conditions for time-dependent interval}

Here, we extend the treatment of the previous section to the case of moving boundaries, by considering time-dependent interval with one moving end which is given as:

$$
x=0 \quad \text { and } \quad x=L(t)
$$

Earlier, the Schrödinger equation with time-dependent boundary conditions given in a one-dimensional box has been studied by many authors (see, e.g., $[12,17]$ and references therein) The Schrödinger equation with timedependent boundary conditions is an ill posed problem unless the moving boundaries are not mapped onto fixed ones $[12,17]$. This can be done by introducing a new coordinate as $[12,17]$ : 


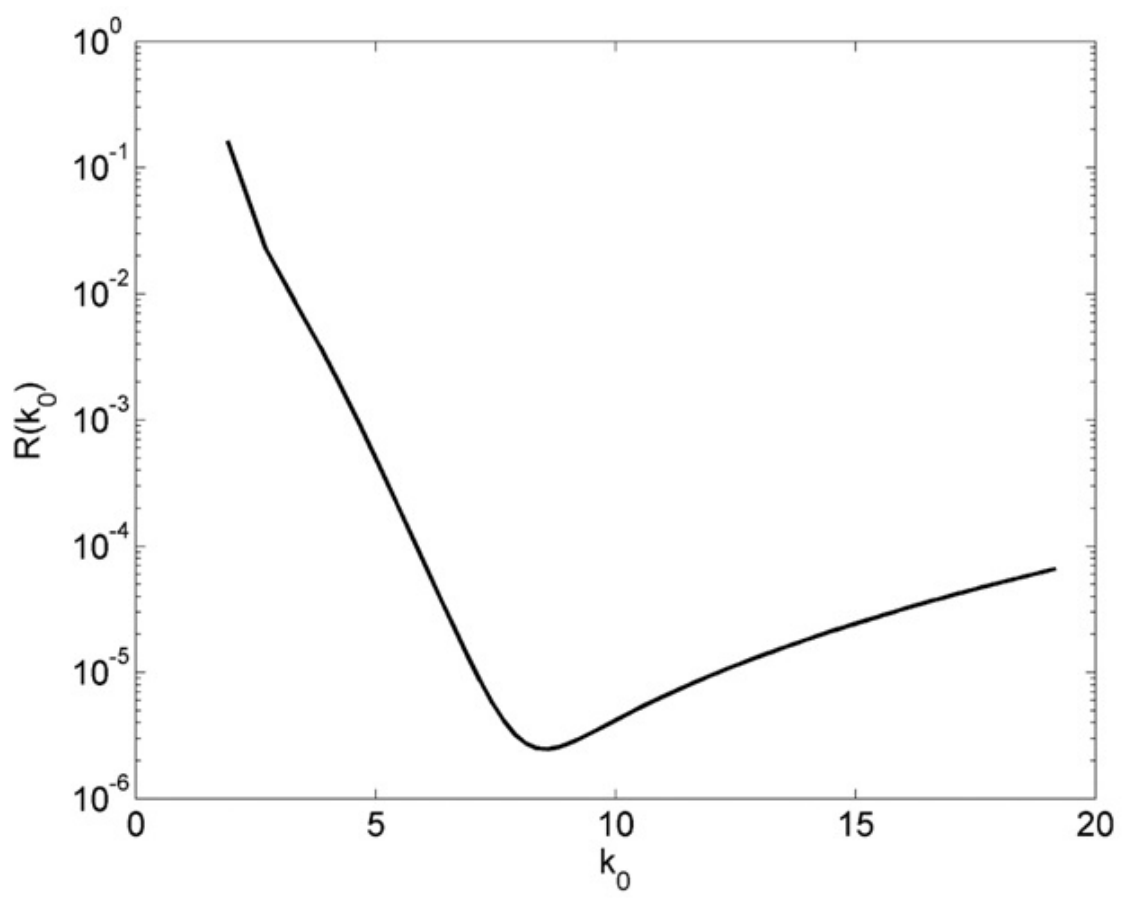

FIG. 2. Dependence of the reflection coefficient on the initial wave vector of wave packet is computed. Initial wave packet width $\sigma=0.05 L$, the length of the interval $L=1$ and the initial position of quantum particle (wave packet) $\xi=3 L / 4$. Numerical calculations are carried out with the discretization parameters: one dimensional space grid size $\Delta x=10^{-3}$ and time step size $\Delta t=10^{-4}$.

$$
y=\frac{x}{L(t)},
$$

which leads to Eq. (1) in the following form $(V=0)$ :

$$
i \frac{\partial \psi(y, t)}{\partial t}=-\frac{1}{2 L^{2}} \frac{\partial^{2} \psi(y, t)}{\partial y^{2}}+i \frac{\dot{L}}{L} y \frac{\partial \psi(y, t)}{\partial t},
$$

with

$$
\dot{L}=\frac{d L}{d t} .
$$

And for the partial differential equation (13), one can apply the new boundary conditions at $y=0$ and $y=1$. The normalization condition for the wave function $\psi(y, t)$ is given by:

$$
\int_{-\infty}^{+\infty} \psi^{*}(y, t) \psi(y, t) d y=\text { const. }
$$

To construct $\mathrm{ABC}$, the boundary should be almost transparent for a plane wave of the form:

$$
\psi(y, t)=\exp (-i \omega t+i k L y)
$$

Then, from Eqs. (15) and (13), one gets the dispersion relation for the wave vector $k$ :

$$
\omega=\frac{k^{2}}{2} \text {. }
$$

This relation can be solved for $k$ and yields:

$$
k^{2}-2 \omega=0,
$$

with the solution:

$$
k_{1,2}= \pm \sqrt{2 \omega} .
$$




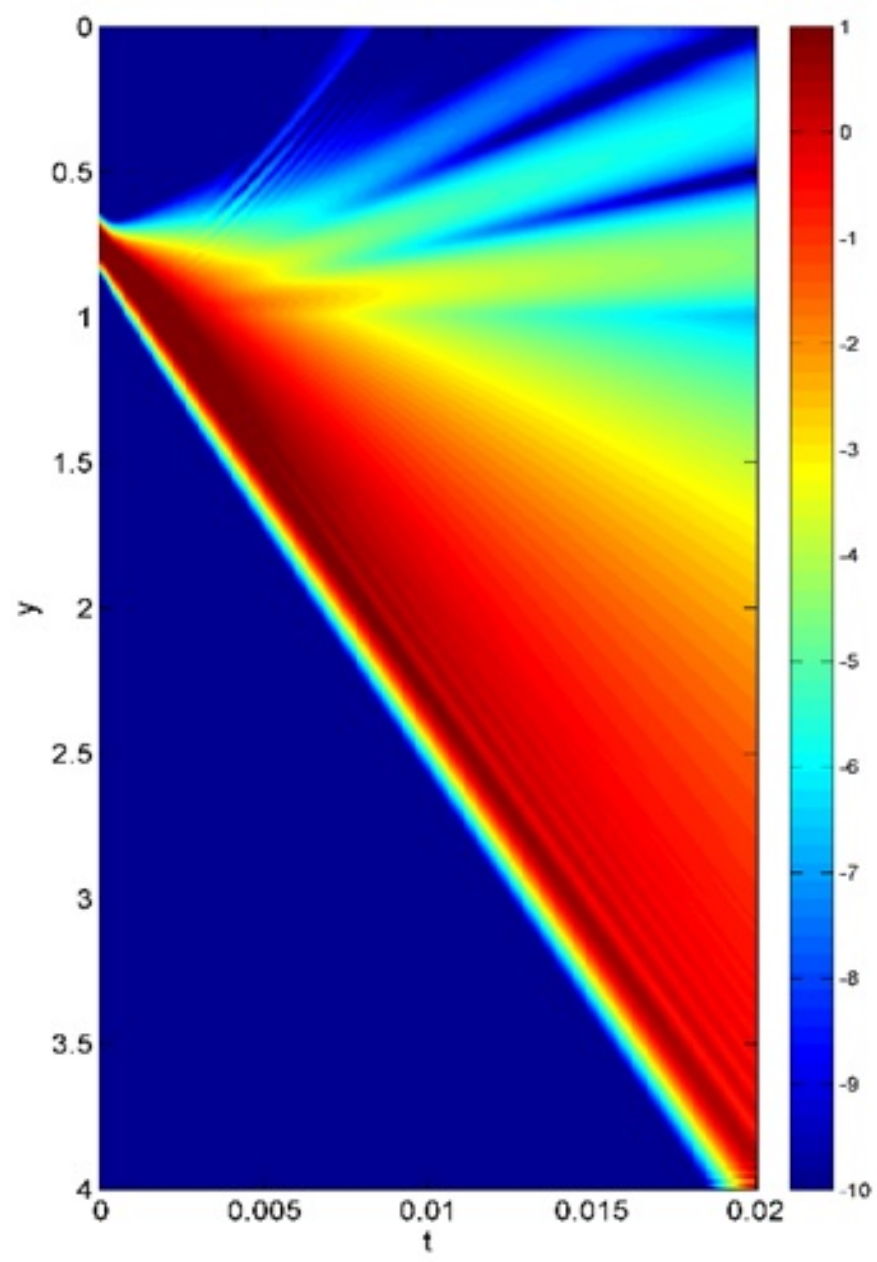

FIG. 3. The spatiotemporal evolution of the probability density in logarithmic scale for the Gaussian wave packet described by Eq. (13) for linearly moving (with velocity $b=10^{-6}$ ) boundary given by Eq. (17). The mean value of wave vector of the initial wave packet $k_{0}=70$. Initial value of the wave packet width $\sigma=0.001 L_{0}$, the initial length of the interval $L_{0}=1$ and the initial position of quantum particle (wave packet) at $\xi=3 L_{0} / 4$. Numerical calculations are carried out with the discretization parameters: one dimensional fixed space grid size $\Delta y=10^{-3}$, and time step size $\Delta t=10^{-4}$.

Using the rational function approximation as in the above equation (5) we write:

$$
k= \pm k_{0} \sqrt{\frac{2 \omega}{k_{0}^{2}}} \approx \pm k_{0} \frac{1+3 z}{3+z}
$$

with

$$
z=\frac{2 \omega}{k_{0}^{2}}
$$

Replacing $k$ and $\omega$ by corresponding differential operators as:

$$
k \Leftrightarrow-i \frac{\partial}{\partial y}, \quad \omega \Leftrightarrow i \frac{\partial}{\partial t},
$$

we obtain adsorbing boundary conditions the same as in the case of a fixed interval Eq. (7):

$$
-\frac{3 i k_{0}^{2}}{2} \frac{\partial}{\partial y} \psi(y, t)+\frac{\partial^{2}}{\partial t \partial y} \psi(y, t)= \pm \frac{k_{0}^{3}}{2} \psi(y, t) \pm 3 i k_{0} \frac{\partial}{\partial t} \psi(y, t) .
$$

Here, the equation with the positive sign corresponds to the $\mathrm{ABC}$ at the right boundary, while the negative sign corresponds to the $\mathrm{ABC}$ at the left boundary. 


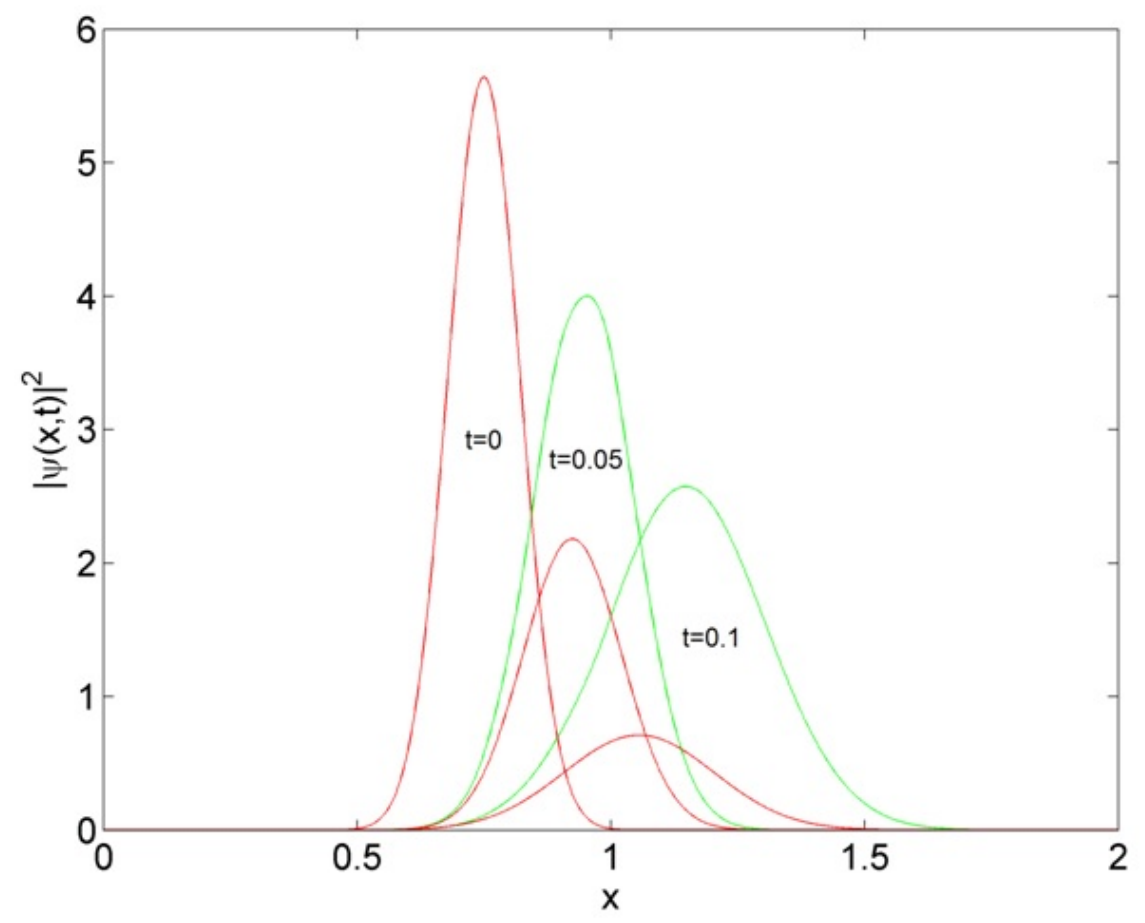

FIG. 4. (Color online) Profile of the Gaussian WP at different time moments for fixed (green) and for time-dependent (red) intervals. The parameters of the packet are chosen as $\xi=3 / 4$; $\sigma=0.001 ; k_{0}=20$.

Furthermore, using the following discretization scheme [11]:

with

$$
\begin{aligned}
\psi(y, t) & \approx \frac{1}{4}\left(\psi_{j+1}^{n+1}+\psi_{j}^{n+1}+\psi_{j+1}^{n}+\psi_{j}^{n}\right), \\
\frac{\partial \psi}{\partial y}(y, t) & \approx \frac{1}{2 \Delta y}\left(\psi_{j+1}^{n+1}-\psi_{j}^{n+1}+\psi_{j+1}^{n}-\psi_{j}^{n}\right), \\
\frac{\partial \psi}{\partial t}(y, t) & \approx \frac{1}{2 \Delta t}\left(\psi_{j+1}^{n+1}+\psi_{j}^{n+1}-\psi_{j+1}^{n}-\psi_{j}^{n}\right), \\
\frac{\partial^{2} \psi}{\partial t \partial y}(y, t) & \approx \frac{1}{\Delta y \Delta t}\left(\psi_{j+1}^{n+1}-\psi_{j}^{n+1}-\psi_{j+1}^{n}+\psi_{j}^{n}\right) .
\end{aligned}
$$

$$
\psi_{j}^{n}=\psi\left(y_{j}, t_{n}\right)
$$

We have the following discretization for the boundary condition:

$$
\begin{aligned}
& -3 i k_{0}^{2} \frac{1}{2 \Delta y}\left(\psi_{j+1}^{n+1}-\psi_{j}^{n+1}+\psi_{j+1}^{n}-\psi_{j}^{n}\right)+\frac{1}{\Delta y \Delta t}\left(\psi_{j+1}^{n+1}-\psi_{j}^{n+1}-\psi_{j+1}^{n}+\psi_{j}^{n}\right) \\
& = \pm k_{0}^{3} \frac{1}{4}\left(\psi_{j+1}^{n+1}+\psi_{j}^{n+1}+\psi_{j+1}^{n}+\psi_{j}^{n}\right) \pm 3 i k_{0} \frac{1}{2 \Delta t}\left(\psi_{j+1}^{n+1}+\psi_{j}^{n+1}-\psi_{j+1}^{n}-\psi_{j}^{n}\right)
\end{aligned}
$$

or

$$
\begin{cases}b_{2} \psi_{j}^{n+1}+b_{1} \psi_{j+1}^{n+1}=b_{4} \psi_{j}^{n}+b_{3} \psi_{j+1}^{n}, & \text { for } \quad y=0,(j=1), \\ b_{1} \psi_{j}^{n+1}+b_{2} \psi_{j+1}^{n+1}=b_{3} \psi_{j}^{n}+b_{4} \psi_{j+1}^{n}, & \text { for } \quad y=1,(j=y \Delta y),\end{cases}
$$

where the coefficients are given:

$$
\begin{aligned}
& b_{1}=-\frac{3 i k_{0}^{2}}{2 \Delta y}+\frac{1}{\Delta y \Delta t}+\frac{k_{0}^{3}}{4}+\frac{3 i k_{0}}{2 \Delta t}, \quad b_{2}=\frac{3 i k_{0}^{2}}{2 \Delta y}-\frac{1}{\Delta y \Delta t}+\frac{k_{0}^{3}}{4}+\frac{3 i k_{0}}{2 \Delta t}, \\
& b_{3}=-\frac{3 i k_{0}^{2}}{2 \Delta y}-\frac{1}{\Delta y \Delta t}+\frac{k_{0}^{3}}{4}-\frac{3 i k_{0}}{2 \Delta t}, \quad b_{4}=\frac{3 i k_{0}^{2}}{2 \Delta y}+\frac{1}{\Delta y \Delta t}+\frac{k_{0}^{3}}{4}-\frac{3 i k_{0}}{2 \Delta t} .
\end{aligned}
$$

In Fig. 3, the evolution of the Gaussian wave packet described by Eq. (13) is presented for the linearly moving boundary given by:

$$
L(t)=L_{0}+b t .
$$


The plot shows reflectionless transmission of the packet through the boundary of the interval.

Figure 4 presents the profile at the GWP at different time moments both for constant and time-dependent intervals. The initial position of the packet is chosen as $\xi=3 / 4$. As is shown in the plot, in case of timedependent $\mathrm{BCs}$ dispersion of the packet occurs more quickly than that for the fixed interval.

\section{Conclusions}

Thus, we have formulated and solved the problem of absorbing boundary conditions for the time-dependent interval by considering time-dependent Schrödinger equation. The time-dependence of the boundary is taken as linear. We found that unlike the case of fixed boundaries, for time-dependent absorbing boundary conditions provide more extensive (reflectionless) transmission of the particle through the domain boundaries and during considerably shorter time. The results of this work can be useful for modeling of quantum systems touching to absorbing or transparent media.

\section{References}

[1] Goldberg A., Schey H.M. and Schwartz J.I. Computer-generated motion pictures of one-dimensional quantum-mechanical transmission and reflection phenomena. Am. J. Phys., 1967, 35(3), P. 177.

[2] Galbraith I., Ching Y.S. and Abraham E. Two dimensional time dependent quantum mechanical scattering event. Am. J. Phys., 1984, 52(1), P.60-68.

[3] Frensley W. Boundary conditions for open quantum systems driven far from equilibrium. Rev. Mod. Phys., $1990,62(3)$, P. 745.

[4] Engquist B. and Majda A. Absorbing boundary conditions for numerical simulation of waves. Math. Comput., 1977, 31(139), P. 629-651.

[5] Givoli D. Non-reflecting boundary conditions. J. Comp. Phys., 1991, 94(1), P. 1-29.

[6] Kosloff R. and Koslof D. Absorbing boundaries for wave propagation problems. J. Comp. Phys., 1986, 63(2), P. $363-376$.

[7] Antoine X. et al. Absorbing boundary conditions for relativistic quantum mechanics equations. J. Comput. Phys., 2014, 277, P. 268-304.

[8] Antoine X. et al. Absorbing boundary conditions for the one-dimensional Schrödinger equation with an exterior repulsive potential. $J$. Comput. Phys., 2009, 228(2), P. 312-335.

[9] Arnold A. Numerically absorbing boundary conditions for quantum evolution equations. VLSI design, 1998, 6(1-4), P. 313-319.

[10] Shibata T. Absorbing boundary conditions for the finite-difference time-domain calculation of the one-dimensional Schrödinger equation. Phys. Rev. B, 1991, 43(11), P. 6760.

[11] Kuska J.-P. Absorbing boundary conditions for the Schrödinger equation on finite intervals. Phys. Rev. B, 1992, 46(11), P. 5000.

[12] Makowski A.J. and Dembiñski S.T. Exactly solvable models with time-dependent boundary conditions. Phys. Lett. A, 1991, 154(5), P. 217-220.

[13] Crank J. and Nicolson P. A practical method for numerical evaluation of solutions of partial differential equations of the heat-conduction type. Advances in Comput. Math., 1996, 6(1), P. 207-226.

[14] Dubeibe F.L. Solving the time-dependent Schrödinger equation with absorbing boundary conditions and source terms in Mathematica 6.0. Int. J. Mod. Phys. C, 2010, 21(14), P. 1391-1406.

[15] Jiang Sh. and Greengard L. Fast evaluation of nonreflecting boundary conditions for the Schrödinger equation in one dimension. Computers \& Mathematics with Applications, 2004, 47(6), P. 955-966.

[16] Neuhasuer D. and Baer M. The time-dependent Schrödinger equation: Application of absorbing boundary conditions. J. Chem. Phys., 1989, 90(11), P. 4351-4355.

[17] Nakamura K. et al. Ideal quantum gas in an expanding cavity: Nature of nonadiabatic force. Phys. Rev. E, 2011, 83(4), P. 041133. 